\title{
GENERALIZED THERMOELASTIC FUNCTIONALLY GRADED HALF SPACE UNDER SURFACE ABSORPTION OF LASER RADIATION
}

\author{
Mohamed N.M. Allam \\ Mansoura University, Faculty of Science, Department of Mathematics, Mansoura, Egypt \\ e-mail:mallam@mans.edu.eg \\ ISMAIL M. TAYEL \\ Majmaah University, College of Education, Department of Mathematics, Saudi Arabia \\ e-mail: i.tayel@mu.edu.sa
}

\begin{abstract}
The subject of this paper is to study the thermoelastic behavior of a functionally graded semi-infinite medium heated uniformly by a laser beam having temporally Gaussian distribution. The surface of the medium is taken as traction free. The general solution is obtained in the Laplace transform domain. The inverse of the Laplace transform is computed numerically using the Riemann-sum approximation method. The numerical results for temperature, displacement and stress are obtained and presented graphically for the generalized theory of thermo-elasticity with one relaxation time.
\end{abstract}

Keywords: laser pulse, generalized thermoelasticity, non-homogeneous medium, numerical inverse of Laplace transform

\section{Introduction}

The study of the problem of thermoelasticity has been taken up by several authors. Biot (1956) developed the coupled theory of thermoelasticity to eliminate the paradox inherent in the classical uncoupled theory according to which elastic changes have no effect on temperature. The main drawback of Biot's equations was that they were based on Fourier's low, which predicted an infinite speed of propagation of heat.

Lord and Shulman (1967) derived equations of dynamic thermoelasticity based on modified Fourier's law, and these equations are usually regarded as the basis of generalized thermoelasticity and called the generalized theory of thermoelasticity with one relaxation time. Green and Lindsay (1972) developed another generalized theory of thermoelasticity. This theory modifies both the energy equation and the Duhamel-Neumann relation. It admits two relaxation times. The theory of thermoelasticity without energy dissipation is another generalized theory and was formulated by Green and Naghdi (1993). It includes the thermal displacement gradient among its independent constitutive variables, and differs from the previous theories in that it does not accommodate dissipation of thermal energy. Ozisik and Tzou (1994), and Tzou (1995a,b) developed a new model called the dual phase-lag model for the heat transport mechanism in which Fourier's law is replaced by an approximation to the modification of Fourier's law with two different time translations for the heat flux and the temperature gradient.

A large amount of work has been devoted to solving thermoelasticity problems with consideration of the coupling effect between temperature and strain rate. Stress waves in a half-space induced by variations of surface strain, temperature or stress were studied by Boley and Tolins (1962) and Chandrasekhariaiah and Srinath (1998). Mozina and Dovc (1994) attempted to use the Laplace transform to solve the thermoelastic stress wave induced by volumetric heating. 
Due to the difficulty in finding analytical Green's functions, only a solution for locations on the surface was obtained.

Researches have also been examining thermoelastic problems with consideration of the non-Fourier effect, but without considering the coupling effect between temperature and strain rate. Kao (1976) was the first to investigate the non-Fourier effect and the thermoelastic wave in a half-space.

When a solid is illuminated with a laser pulse, absorption of the pulse leads to a localized temperature increase which in turn causes thermal expansion and generates a thermoelastic wave in the solid (Wang and $\mathrm{Xu}, 2001$ ). McDonald (1990) studied the importance of thermal diffusion on the generation of thermoelastic waves in metals induced by surface Gaussian laser beam heating. Engelhard and Bertrand (1977) studied the influence of optical penetration depth and the laser pulse duration on longitudinal acoustic waves induced by volumetric absorption of a laser beam. Henain et al. (2014) studied the thermoelastic interaction caused by heating a homogeneous and isotropic thermoelastic semi-infinite body induced by a Gaussian laser pulse. Allam et al. (2014) studied thermoelastic waves induced by pulsed laser in a non-homogeneous microscal beam.

The purpose of the present work is to study the thermoelastic interaction caused by heating a non-homogeneous and isotropic thermoelastic semi-infinite body induced by a laser pulse by employing the generalized theory of thermoelasticity. The problem is solved by using Laplace transform techniques. The inverse Laplace transform is computed numerically using the Riemann-sum approximation method. Numerical solutions for spatial temperature, displacement and stress are obtained using the generalized theory of thermoelasticity with one relaxation time. At the end of this work, we present the computed results obtained from the theoretical relations applied on a $(\mathrm{Cu})$ target.

\section{Mathematical modeling and basic equations}

Consider a non-homogeneous anisotropic thermally conducting elastic solid at a uniform temperature $T_{0}$. The governing equations for linear generalized thermoelastic media, in the absence of incremental body forces, and heat source are discussed below:

- The general model of heat conduction equation corresponding to five models of thermoelasticity takes the form (Allam et al., 2014)

$$
\left(1+\lambda_{2 k} \frac{\partial}{\partial t}\right)\left(K_{i j} \theta_{j}\right)_{, i}=\left(\lambda_{3 k}+\lambda_{4 k} \frac{\partial}{\partial t}+\lambda_{5 k} \frac{\partial^{2}}{\partial t^{2}}\right)\left(\rho C_{E} \frac{\partial \theta}{\partial t}+T_{0} \beta_{i j} \frac{\partial u_{m, m}}{\partial t}\right)
$$

- The constitutive equations for an anisotropic medium are given by

$$
\sigma_{i j}=C_{i j m n} \varepsilon_{m n}-\beta_{i j} \delta_{i j}\left(1+\lambda_{1 k} \frac{\partial}{\partial t}\right) \theta \quad \begin{aligned}
& i, j=1,2,3 \\
& k=1,2, \ldots, 5
\end{aligned}
$$

The parameters $\lambda_{i k}$, are given by

$$
\lambda_{i k}=\left[\begin{array}{ccccc}
0 & 0 & \tau_{1} & 0 & 0 \\
0 & 0 & 0 & 0 & \tau_{\theta} \\
1 & 1 & 1 & 0 & 1 \\
0 & \tau_{0} & \tau_{0} & 1 & \tau_{q} \\
0 & 0 & 0 & 0 & \frac{1}{2} \tau_{q}^{2}
\end{array}\right]
$$

which may be called the relaxation time matrix, or given by the relations

$$
\begin{aligned}
& \lambda_{1 k}=\tau_{1} \delta_{3 k} \quad \lambda_{2 k}=\tau_{\theta} \delta_{5 k} \quad \lambda_{3 k}=1-\delta_{4 k} \\
& \lambda_{4 k}=\delta_{4 k}+\tau_{0}\left(\delta_{2 k}+\delta_{3 k}\right)+\tau_{q} \delta_{5 k} \quad \lambda_{5 k}=\frac{1}{2} \tau_{q}^{2} \delta_{5 k}
\end{aligned}
$$


- The strain-displacement relations are given by Cauchy's relations

$$
\varepsilon_{i j}=\frac{1}{2}\left(u_{i, j}+u_{j, i}\right)
$$

- The equations of motion are

$$
C_{i j m n} u_{m, j n}-\beta_{i j}\left(1+\lambda_{1 k} \frac{\partial}{\partial t}\right) \theta_{, j}=\rho \ddot{u}_{i}
$$

where $C_{i j m n}$ are isothermal elastic constants, $u_{i}$ are the displacement components, $\theta=T-T_{0}$ is the temperature increment, and $T_{0}$ is the environmental temperature assumed to be such that $\left|\theta / T_{0}\right| \ll 1$.

In the above formulas, $K_{i j}$ is the thermal conductivity tensor, $C_{E}$ - specific heat at a constant strain, $\tau_{0}, \tau_{1}$ are the 1st and 2nd relaxation times, $\tau_{q}$ is the phase-lag of the heat flux, $\tau_{\theta}$ is the phase-lag of the temperature gradient $0 \leqslant \tau_{\theta}<\tau_{q}$ and $\rho$ is the mass density. The dummy index implies summation. The dot and comma notations denote differentiation with respect to time and space, respectively.

Equations (2.1)-(2.4) describe the coupled dynamical thermoelasticity theory, generalized thermoelasticity theory proposed by Lord and Shulman, generalized thermo-elasticity theory with two relaxation times developed by Green and Lindsay, Green and Naghdi theory without energy dissipation and dual phase-lag model for different sets of values of the parameters $\lambda_{i k}$.

In equations (2.1)-(2.4):

1 ) if we put $k=1$, then they reduce to equations of the classical theory of thermoelasticity $(\mathrm{CTE})$

2) when $k=2$, then they reduce to equations of the generalized theory with one relaxation time (LS),

3) putting $k=3$, then they reduce to equations of the generalized theory with two relaxation time (GL),

4) when $k=4$ and $K=K^{*}$ ( $K^{*}$ is a material constant characteristic of the Green and Naghdi theory), then they reduce to equations of the generalized theory without energy dissipation (GN).

5) if $k=5$, then they reduce to equations of the generalized theory with dual-phase-lags (DPL).

Consider a thermoelastic, non-homogeneous isotropic semi-infinite medium occupying the region $(z \geqslant 0)$ and initially at uniform temperature $T_{0}$. The surface of the target $(z=0)$ is uniformly heated by a pulsed laser beam and assumed to be traction free. The Cartesian coordinates $(x, y, z)$ are considered in the solution with $\mathrm{z}$-axis pointing vertically into the medium.

The generalized equation of heat conduction (2.1) takes the form

$$
\left(1+\lambda_{2 k} \frac{\partial}{\partial t}\right)\left(\nabla K \cdot \nabla \theta+K \nabla^{2} \theta\right)=\left(\lambda_{3 k}+\lambda_{4 k} \frac{\partial}{\partial t}+\lambda_{5 k} \frac{\partial^{2}}{\partial t^{2}}\right) \frac{\partial}{\partial t}\left(\rho C_{E} \theta+\gamma T_{0} e\right)
$$

where $\gamma=E \alpha_{t} /(1-2 \nu), E$ is Young's modulus, $\alpha_{t}$ is the thermal expansion coefficient, $\nu$ is Poisson's ratio, $e$ is the relative volume dilatation and $\nabla^{2}$ is the Laplace operator.

The equations of motion in the case of body free forces (2.4) reduces to

$$
\frac{\partial \sigma_{z z}}{\partial z}=\rho \frac{\partial^{2} w(z, t)}{\partial t^{2}}
$$

$w(z, t)$ is the only component of the displacement vector. 
The strain components (2.3) become

$$
e_{z z}=\frac{\partial w}{\partial z} \quad e_{x x}=e_{y y}=e_{x y}=e_{x z}=e_{y z}=0
$$

The volume dilatation e and Laplace operator are thus given by

$$
e=e_{x x}+e_{y y}+e_{z z}=\frac{\partial w}{\partial z} \quad \nabla^{2}=\frac{\partial^{2}}{\partial z^{2}}
$$

Using (2.7) and (2.2), the stress components are

$$
\begin{aligned}
\sigma_{z z} & =\frac{E}{1-2 \nu}\left[\frac{1-\nu}{1+\nu} \frac{\partial w}{\partial z}-\left(1+\lambda_{1 k} \frac{\partial}{\partial t}\right) \alpha_{t} \theta\right] \\
\sigma_{x x} & =\sigma_{y y}=\frac{E}{1-2 \nu}\left(\frac{\nu}{1+\nu} \frac{\partial w}{\partial z}-\left(1+\lambda_{1 k} \frac{\partial}{\partial t}\right) \alpha_{t} \theta\right]
\end{aligned}
$$

The boundary conditions at $z=0$

$$
\begin{aligned}
& -\left(1+\lambda_{2 k} \frac{\partial}{\partial t}\right) K \nabla \theta=\left(\lambda_{3 k}+\lambda_{4 k} \frac{\partial}{\partial t}+\lambda_{5 k} \frac{\partial^{2}}{\partial t^{2}}\right) q \\
& \sigma_{z z}=0
\end{aligned}
$$

where $q$ is the laser radiation propagating in the $z$ direction, given by $q=A_{0} q_{0} f(t)$, with $A_{0}$ the coefficient of heat absorption, $q_{0}$ intensity of the laser beam and $f(t)$ temporal distribution of the laser radiation.

We use, sience the material is consiered inhomogeneous

$$
\{E(z), K(z), \rho(z)\}=\left\{E_{0}, K_{0}, \rho_{0}\right\} F(z)
$$

where $E_{0}, K_{0}$ and $\rho_{0}$ are assumed to be constants.

Thus, the stress components are in form

$$
\begin{aligned}
\sigma_{z z} & =\frac{E_{0} F(z)}{1-2 \nu}\left[\frac{1-\nu}{1+\nu} \frac{\partial w}{\partial z}-\left(1+\lambda_{1 k} \frac{\partial}{\partial t}\right) \alpha_{t} \theta\right] \\
\sigma_{x x} & =\sigma_{y y}=\frac{E_{0} F(z)}{1-2 \nu}\left[\frac{\nu}{1+\nu} \frac{\partial w}{\partial z}-\left(1+\lambda_{1 k} \frac{\partial}{\partial t}\right) \alpha_{t} \theta\right]
\end{aligned}
$$

\section{Non-dimensionalization}

The governing equations takes a more convenient form by using the following non-dimensional variables (Allam et al., 2014)

$$
\begin{array}{llll}
\left(z^{\prime}, w^{\prime}\right)=\frac{(z, w)}{h} & c_{0}=\sqrt{\frac{E_{0}}{\rho_{0}}} & \theta^{\prime}=\frac{\theta}{T_{0}} & \\
\sigma_{z}^{\prime}=\frac{\sigma_{z}}{E_{0}} & t^{\prime}=\frac{c_{0}}{h} t & \tau_{i}^{\prime}=\frac{c_{0}}{h} \tau_{i} & i=0,1, q, \theta, p
\end{array}
$$

For simplicity, we drop the dashes of all variables and parameters.

The equation of heat conduction (2.5) is in the form

$$
\left(1+\lambda_{2 k} \frac{\partial}{\partial t}\right)\left(\frac{\partial^{2}}{\partial z^{2}}+\Phi(z) \frac{\partial}{\partial z}\right) \theta=\left(\lambda_{3 k}+\lambda_{4 k} \frac{\partial}{\partial t}+\lambda_{5 k} \frac{\partial^{2}}{\partial t^{2}}\right) \frac{\partial}{\partial t}\left(c_{1} \theta+c_{2} \frac{\partial w}{\partial z}\right)
$$


Equation of motion (2.6) reduces to

$$
c_{3}\left(\frac{\partial^{2}}{\partial z^{2}}+\Phi(z) \frac{\partial}{\partial z}-\frac{1}{c_{3}} \frac{\partial^{2}}{\partial t^{2}}\right) w-c_{4}\left(1+\lambda_{1 k} \frac{\partial}{\partial t}\right)\left(\frac{\partial}{\partial z}+\Phi(z)\right) \theta=0
$$

where

$$
\begin{array}{lll}
c_{1}=\frac{h \rho_{0} C_{E} c_{0}}{K_{0}} & c_{2}=\frac{\alpha_{t} h C_{E} c_{0}}{K_{0}(1-2 \nu)} & c_{3}=\frac{1-\nu}{(1-2 \nu)(1+\nu)} \\
c_{4}=\frac{\alpha_{t} T_{0}}{1-2 \nu} & \Phi(z)=\frac{1}{F(z)} \frac{d F(z)}{d z} &
\end{array}
$$

$z$ is the dimensionless coordinate.

The structure of the governing equations and the boundary conditions suggests the idea of looking for the solution to the problem in a Laplace transform integral, which was given by (Roberts and Kaufman, 1966)

$$
\bar{f}(z, s)=\int_{0}^{\infty} f(z, t) e^{-s t} d t
$$

Thus, equations (3.1) and (3.2) assume the form

$$
\begin{aligned}
& \left(\frac{d^{2}}{d z^{2}}+\Phi(z) \frac{d}{d z}-\Lambda c_{1}\right) \bar{\theta}-\Lambda c_{2} \frac{d \bar{w}}{d z}=0 \\
& c_{3}\left(\frac{d^{2}}{d z^{2}}+\Phi(z) \frac{d}{d z}-\frac{s^{2}}{c_{3}}\right) \bar{w}-c_{4}\left(1+\lambda_{1 k} s\right)\left(\frac{d}{d z}+\Phi(z)\right) \bar{\theta}=0
\end{aligned}
$$

Eliminating $\bar{w}$ or $\bar{\theta}$ from Eqs. (3.4), we arrive at two differential equations for $\bar{\theta}$ and $\bar{w}$

$$
\left(D^{4}+b_{3} D^{3}+b_{2} D^{2}+b_{1} D+b_{0}\right)\{\bar{\theta}, \bar{w}\}=0
$$

which may be written in form

$$
\left[\left(D^{2}+\Phi(z) D\right)^{2}-a\left(D^{2}+\Phi(z) D\right)+\frac{s^{2} \Lambda c_{1}}{c_{3}}-c_{5} \frac{d \Phi(z)}{d z}\right]\{\bar{\theta}, \bar{w}\}=0
$$

where

$$
\begin{aligned}
c_{5} & =\frac{\Lambda c_{2} c_{4}\left(1+\lambda_{1 k} s\right)}{c_{3}} & D & =\frac{d}{d z} \\
b_{0} & =\frac{\Lambda c_{1} s^{2}}{c_{3}}-c_{5} \frac{d \Phi(z)}{d z} & b_{1} & =\left(\frac{d^{2}}{d z^{2}}+\Phi(z) \frac{d}{d z}-a\right) \Phi(z) \\
b_{2} & =\Phi(z)^{2}+2 \frac{d \Phi(z)}{d z}-a & b_{3} & =2 \Phi(z) \\
\Lambda & =\frac{s\left(\lambda_{3 k}+\lambda_{4 k} s+\lambda_{5 k} s^{2}\right)}{1+\lambda_{2 k} s} & a & =\Lambda c_{1}+c_{5}+\frac{s^{2}}{c_{3}}
\end{aligned}
$$

Dimentionless boundary conditions (2.10) after using the Laplace transform are

$$
\left.\frac{d \bar{\theta}}{d z}\right|_{z=0}=-\left.\frac{c_{1} A_{0} q_{0} \Lambda}{s} f(s) \quad \bar{\sigma}_{z z}\right|_{z=0}=0
$$

Equations (3.6) and (3.7) are a complete system of ordinary differential equtions in $\bar{w}$ and $\bar{\theta}$. 


\section{Special case}

We take $F(z)$ in the form $F(z)=e^{-z}$, then $\Phi(z)=(1 / F(z))(d F(z) / d z)=-1$ and then

$$
b_{0}=\frac{\Lambda c_{1} s^{2}}{c_{3}} \quad b_{1}=a \quad b_{2}=1-a \quad b_{3}=-2
$$

In this case, equations (3.6) may be rewritten in form

$$
\left[\left(D^{2}-D\right)^{2}-a\left(D^{2}-D\right)+\frac{s^{2} \Lambda c_{1}}{c_{3}}\right]\{\bar{\theta}, \bar{w}\}=0
$$

Consider the solution to equations (4.2) in form

$$
\bar{\theta}=\sum_{j=1}^{2} A_{j} e^{-m_{j} z} \quad \bar{w}=\sum_{j=1}^{2} B_{j} e^{-m_{j} z}
$$

where $m_{j}(i=1,2)$ are the roots of equation

$$
\left(m^{2}-m\right)^{2}-a\left(m^{2}-m\right)+\frac{s^{2} \Lambda c_{1}}{c_{3}}=0
$$

Substitution of (4.3) into (3.4) 1 , yields

$$
B_{j}=-\frac{m_{j}^{2}+m_{j}-\Lambda c_{1}}{\Lambda c_{2} m_{j}} A_{j} \quad j=1,2
$$

Then, Eqs. (4.3) take the form

$$
\bar{\theta}=\sum_{j=1}^{2} A_{j} e^{-m_{j} z} \quad \bar{w}=\sum_{j=1}^{2}-\frac{m_{j}^{2}+m_{j}-\Lambda c_{1}}{\Lambda c_{2} m_{j}} A_{j} e^{-m_{j} z}
$$

To evaluate the unknown parameters $A_{j}$, we shall use boundary conditions (3.7).

Taking Laplace's transformation of the component of stress $\sigma_{z z}$ in non-dimensional form, yields

$$
\sigma_{z z}=\frac{F(z)}{1-2 \nu}\left[\frac{1-\nu}{1+\nu} \frac{\partial \bar{w}}{\partial z}-\left(1+\lambda_{1 k} s\right) \alpha_{t} T_{0} \bar{\theta}\right]
$$

After applying the boundary conditions, we arrive at the following two equations

$$
\sum_{j=1}^{2} m_{j} A_{j}=-\frac{c_{1} A_{0} q_{0} \Lambda}{s} f(s) \quad \sum_{j=1}^{2} f_{j}(s) A_{j}=0
$$

where

$$
f_{i}(s)=\frac{1-\nu}{1+\nu}\left(\frac{m_{j}^{2}+m_{j}-\Lambda c_{1}}{\Lambda c_{2}}\right)-\left(1+\lambda_{1 k} s\right) \alpha_{t} T_{0} \quad i=1,2
$$

Thus, the solution to system of equations (4.8) is

$$
A_{1}=\frac{\Lambda A_{0} c_{1} q_{0} f(s) f_{2}}{s\left(m_{1} f_{2}+m_{2} f_{1}\right)} \quad A_{2}=-\frac{\Lambda A_{0} c_{1} q_{0} f(s) f_{1}}{s\left(m_{1} f_{2}+m_{2} f_{1}\right)}
$$




\section{Inverse Laplace transform}

Since the formulas of temperature, displacement and stresses are difficult to invert to the time domain, therefore a numerical inverse will be used. In order to invert the Laplace transform of temperature, displacement and stresses, we applay the Riemann-sum approximation method by using the relation

$$
f(t)=\frac{e^{k t}}{t}\left[\frac{1}{2} \bar{f}(k)+\operatorname{Re} \sum_{n=1}^{N}(-1)^{n} \bar{f}\left(k+\frac{\mathrm{i} n \pi}{t}\right)\right]
$$

where Re is the real part, $\mathrm{i}=\sqrt{-1}$ is the imaginary unit number and $N$ is a sufficiently large integer representing the number of terms. For faster convergence, numerous numerical experiments have shown that the value of $k$ should satisfy the relation $k t=4.7$, see (Tzou, 1995a,b).

\section{Application and computation}

Now the generalized theory of thermoelasticity with one relaxation time (LS), where $k=2$ will be considered in the calculation. Consider the intensity of the laser pulse to be given by a Gaussian distribution $G(t)=\exp \left[-\left(t-t_{0}\right)^{2} /(\Delta t)^{2}\right]$ that the laser beam incidents uniformly on a (Copper) target. Temperature, displacement and stresses are to be calculated taking the following constants (Henain et al., 2014)

$$
\begin{array}{lll}
T_{0}=293 \mathrm{~K} & \rho_{0}=8954 \mathrm{~kg} / \mathrm{m}^{3} & \tau_{0}=35 \cdot 10^{-15} \mathrm{~s} \\
c_{E}=383.1 \mathrm{~J} /(\mathrm{kg} \cdot \mathrm{K}) & \alpha_{t}=1.78 \cdot 10^{-5} \mathrm{~K}^{-1} & k_{0}=386 \mathrm{~W} /(\mathrm{m} \cdot \mathrm{K}) \\
t_{0}=3 \cdot 10^{-3} \mathrm{~s} & \Delta t=10^{-3} \mathrm{~s} & E_{0}=89.6 \mathrm{GPa} \\
\nu=0.44 & h=1 \mathrm{~m} & A_{0}=0.01
\end{array}
$$

where $t_{0}$ is the time at which $G(t)$ is maximum, $\Delta t$ is the time at which the intensity of the laser beam reduces to $1 / e$.

\section{Results and discussion}

Figure 1 represents in curve (a) the laser pulse with the maximum value at $t=0.003$. The surface temperature distribution calculated per unit intensity is represented in curve (b). From the figure, it is evident that the maximum of the temperature distribution occurs at a time greater than the maximum of the laser pulse. This behavior can be attributed to the fact that at the beginning of the laser pulse the absorbed power compensates the heat losses due to conductivity. This fact leads to an increase in temperature. This increase lasts up until the absorbed laser power is equal to the heat losses, when the maximum of the temperature occurs. After this point, the absorbed laser radiation can not compensate the losses, and the temperature begins to decrease.

Figure 2 represents the temperature distribution per unit intensity calculated at different times as a function of $z$. In Fig. 2, it is shown that the temperature decreases as $z$ increases, and that for $(t=0.002, t=0.004$ and $t=0.005)$ it is smaller than for $(t=0.0035)$. This can be attributed to the temporal profile of the laser radiation which is chosen to be Gaussian having its peak value at $(0.003)$ and a half width $(0.003)$. It is noted that the temperature moves deeper in the target as the time increases and vanishes at large values of $z$. 


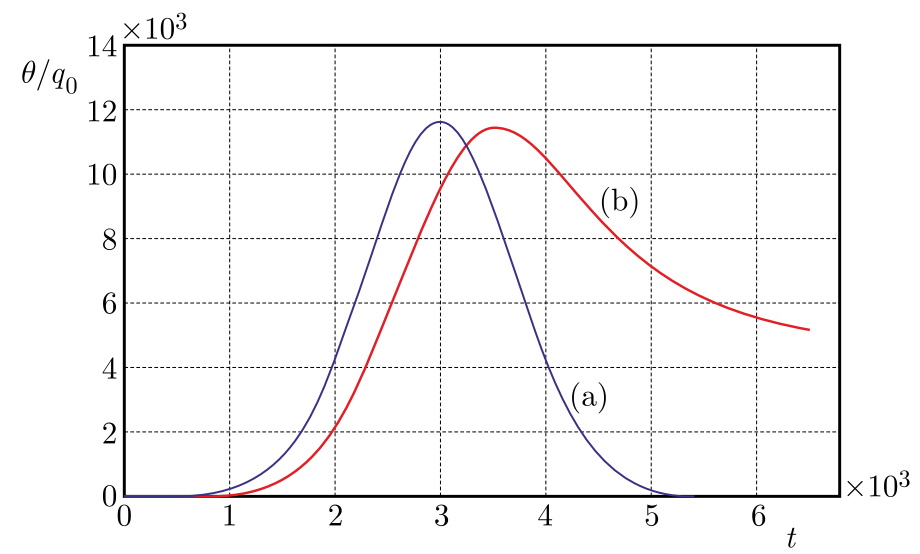

Fig. 1. (a) Temporal behavior of the laser radiation, (b) surface temporal temperature distribution per unit intensity

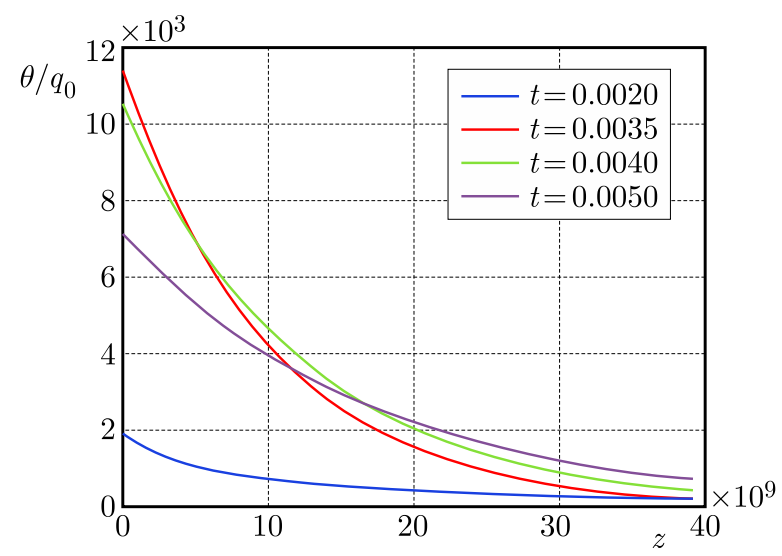

Fig. 2. Temperature distribution per unit intensity as a function of $z$ for different time parameters

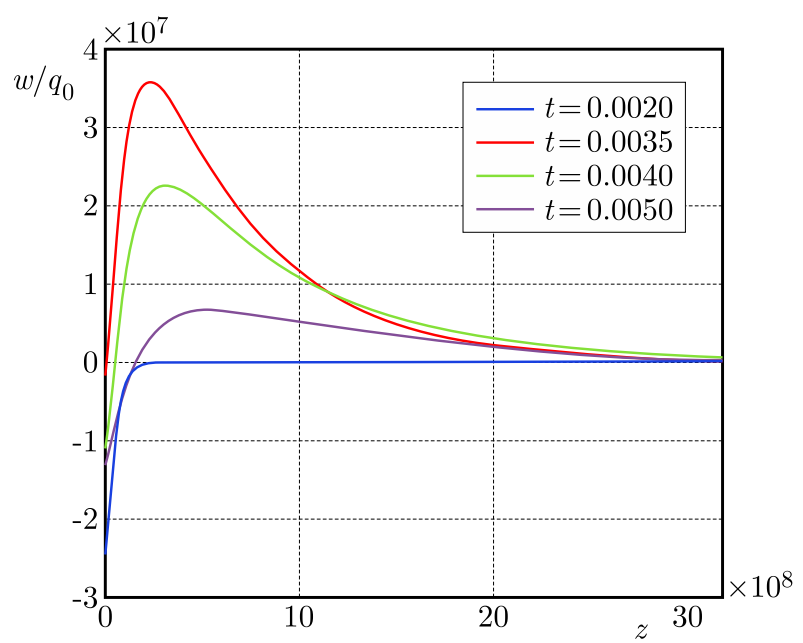

Fig. 3. Displacement distribution per unit intensity as a function of $z$ for different time parameters

Figure 3 represents the displacement $w$ calculated for different $z$ values with time as the parameter. It is found that it is a negative displacement at $z=0$ and its vicinity, which is due to the heating effect of laser radiation that allowed the particles to move in the upward direction in the free half space corresponding to negative values of $z$. By increasing $z$ values, Fig. 3 shows a positive displacement representing also movement of the particles downward. 
Figure 4 represents the calculated spatial stress $\sigma_{z z}$ per unit intensity calculated at different $z$ values with time as the parameter. Figure 4 shows a strong negative gradient decrease with an increase in the $z$ values. It takes place after reaching their maximum magnitude when the positive gradient decreases with increasing $z$ values. It is also seen that the behavior of the curve with time is followed by the chosen temporal behavior of the laser radiation.

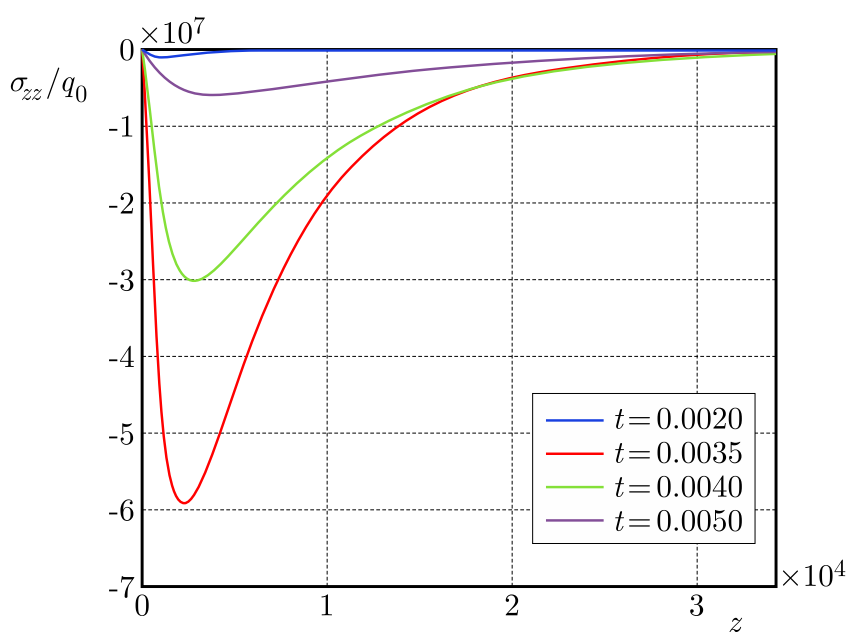

Fig. 4. Stress distribution $\sigma_{z z}$ per unit intensity as a function of $z$ for different time parameters

Figures 5 represents $\sigma_{x x}$ per unit intensity as a function of $z$ with time as the parameter. The curves show the same behavior as $\sigma_{z z}$.

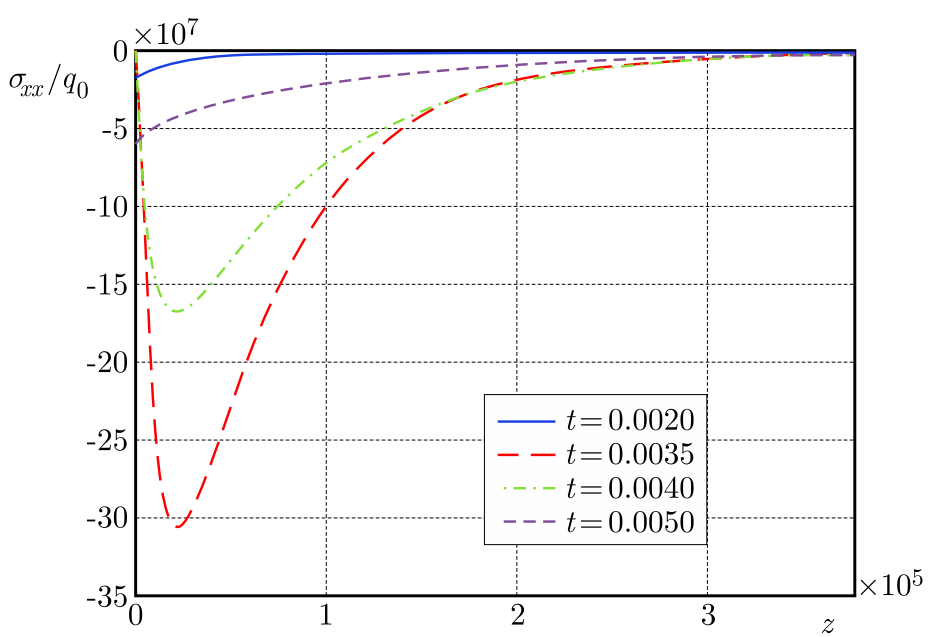

Fig. 5. Stress distribution $\sigma_{x x}$ per unit intensity as a function of $z$ for different time parameters

Figure 6 represents the temperature distribution $\theta$ per unit intensity as a function of $z$ at the time $(t=0.0035)$ with $h$ as the parameter. It is seen that for a fixed time and different values of $h$, the temperature decreases and enlarges $z$ values as $h$ increases.

Figure 7 represents the displacement distribution $w$ per unit intensity as a function of $z$ at the time $(t=0.0035)$ with $h$ as the parameter. One can observe that for a fixed time and different values of $h$, the displacment decreases and goes down to small $z$ value as $h$ increases. 


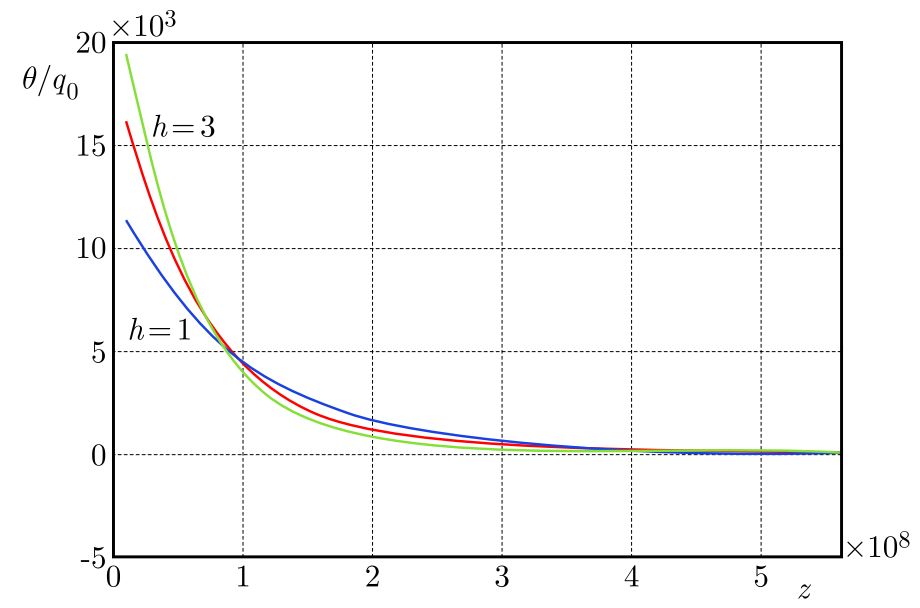

Fig. 6. Temperature distribution $\theta$ per unit intensity as a function of $z$ at $t=0.0035$ with $h$ as the parameter

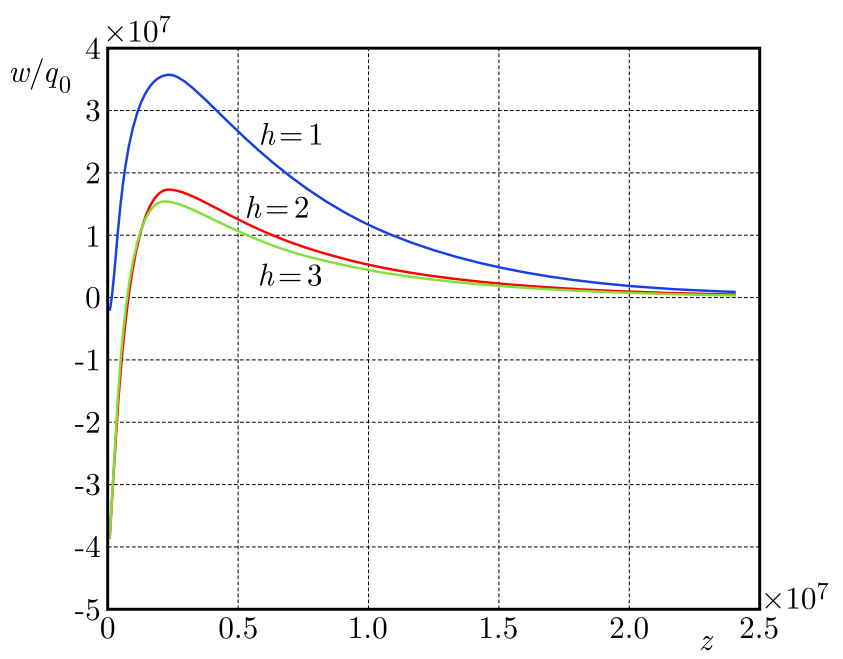

Fig. 7. Displacement distribution $w$ per unit intensity as a function of $z$ at $t=0.0035$ with $h$ as the parameter

\section{References}

1. Allam M.N.M., Abouelregal A.E., 2014, The thermoelastic waves induced by pulsed laser and varying heat of inhomogeneous microscale beam resonators, Journal of Thermal Stresses, 37, 4, 455-470

2. Biот M.A., 1956, Thermoelasticity and irreversible thermodynamics, Journal of Applied Physics, 27, 3, 240-253

3. Boley B.A., Tolins I.S., 1962, Transient coupled thermoelastic boundary value problems in the half-space, Journal of Applied Mechanics, 29, 637-646

4. Chandrasekharaiah D.S., Srinath K.S., 1998, Thermoelastic interactions without energy dissipation due to a point heat source, Journal of Elasticity, 5, 97-108

5. Enguehard F., Bertrand L., 1977, Effects of optical penetration and laser pulse duration on laser generated longitudinal acoustic waves, Journal of Applied Physics, 82, 1532-1538

6. Green A.E., Lindsay K.A., 1972, Thermoelasticity, Journal of Elasticity, 2, 1-7

7. Green A.E., NAghdi P.M., 1993, Thermoelasticity without energy dissipation, Journal of Elasticity, 31, 189-208 
8. Henain E.F., Hassan A.F., Megahed F., Tayel I.M., 2014, Thermoelastic half space under illumination of a laser beam using lord and shulman theory, Journal of Thermal Stresses, 37, 51-72

9. KAO T.T., 1976, On thermally induced non-fourier stress waves in a semi-infinite medium, $A I A A$ Journal, 14, 818-820

10. Lord, H.W. And Shulman, Y., 1967, A Generalized dynamical theory of thermoelasticity, Journal of the Mechanics and Physics of Solids, 15, 299-309

11. MCDonald F.A., 1990, On the precursor in laser-generated ultrasound wave forms in metals, Journal of Applied Physics Letters, 56, 230-232

12. Mozina J., Dovc M., 1994, One-dimensional model of optically induced thermoelastic waves, Modern Physics Letters, 8, 1791-1801

13. OzISIK M.N., Tzou D.Y., 1994, On the wave theory of heat conduction, Journal of Heat Transfer (ASME), 116, 526-535

14. Roberts G.E., Kaufman H., 1966, Table of Laplace Transform, W.B. Saunders, Philadelphia

15. Tzou D.Y., 1995a, Experimental support for the lagging behavior in heat propagation, Journal of Thermophysics and Heat Transfer, 9 , 686-693

16. Tzou D.Y., 1995b, A unified approach for heat conduction from macro to micro scales, Journal of Heat Transfer (ASME), 117, 8-16

17. Wang X., Xu X., 2001, Thermoelastic wave induced by pulsed laser heating, Journal of Applied Physics, 73, 107-114

Manuscript received February 7, 2016; accepted for print June 18, 2016 